\title{
ReSEARCH
}

\section{PET in Halifax}

Jonathan Chung', Colin Van Zoost ${ }^{2}$, Steve Burrell ${ }^{3}$

${ }^{1}$ Class of 2011, Faculty of Medicine, Dalhousie University

2Internal Medicine, Dalhousie University

${ }^{3}$ Department of Diagnostic Radiology, Dalhousie University, QEII Health Sciences Centre

\begin{abstract}
With the recent installation of a PET-CT scanner in Halifax, local clinicians may now take advantage of PET's various applications in the field of oncology, where it has become a standard of care. PET also plays a role in the areas of cardiology and neurology although it has not yet been approved for these uses in Nova Scotia. This article describes the basic principles of PET and radiopharmaceuticals as well as PET's increasing role in the management of patients in the areas of oncology, neurology and cardiology. PET shows significant promise in these areas and clinicians should be familiar with its many applications.
\end{abstract}

\section{Introduction}

Positron Emission Tomography (PET) is a state of the art diagnostic imaging tool with a wide range of applications in oncology, cardiology and neurology. Distinct from other imaging modalities such as computed tomography (CT), magnetic resonance imaging (MRI) and ultrasound that use anatomical characteristics as their major diagnostic criteria, PET exploits the metabolic properties of cells to allow imaging at the molecular level. The greatest clinical indication for PET, representing $85-90 \%$ of current utilization worldwide, is in oncology. In a wide variety of cancers a PET scan can evaluate the entire body to give a comprehensive staging of the cancer, aid in radiation therapy planning, monitor the success of therapy, and assess for disease recurrence. Furthermore, in certain patients without a known cancer, PET is useful in distinguishing whether a newly discovered mass is benign or malignant.

PET imaging also plays important niche roles in neurology, where it is used to assess dementias, movement disorders, and seizures, and in cardiology, where it is used to assess myocardial viability and perfusion. In addition to these important clinical applications, PET imaging can play a valuable role in a number of research applications.

\section{Physics}

PET technology relies on the release of positively charged anti-electrons called positrons from radiopharmaceuticals (tracers), which have been injected into the patient and have localized to a disease process such as cancer. Positron emission takes place when an unstable nucleus with an excess positive change emits a positron to become stable. A short distance from where the positron is emitted from its source (for example $2-3 \mathrm{~mm}$ for the fluorine radioisotope ${ }^{18} \mathrm{~F}$ ) it loses energy and interacts with an electron, resulting in annihilation of both the positron and the electron. During annihilation, the masses are converted into two gamma rays, or photons, that travel in a 180 degree path opposite to one another. Two detectors then perceive these photons at virtually the same time, in a process termed coincidence. Thus, when coincidence is detected it is known that the annihilation must have taken place somewhere along the line between the detectors. By recording all the coincidences within a 360 degree field around the patient, a 3 dimensional image can be constructed, reflecting the distribution of the radiopharmaceutical throughout the body.

\section{Radiopharmaceuticals}

In nuclear medicine, which includes PET, the tracers used are called radiopharmaceuticals. Radiopharmaceuticals consist of two components, the first of which is the radioisotope which is the radioactive component that undergoes decay, releasing the photons that are detected by the camera. The second component is the pharmaceutical, which is the component possessing the desired physiologic property that results in the radiopharmaceutical localizing to the disease process. The major radioisotopes used in 


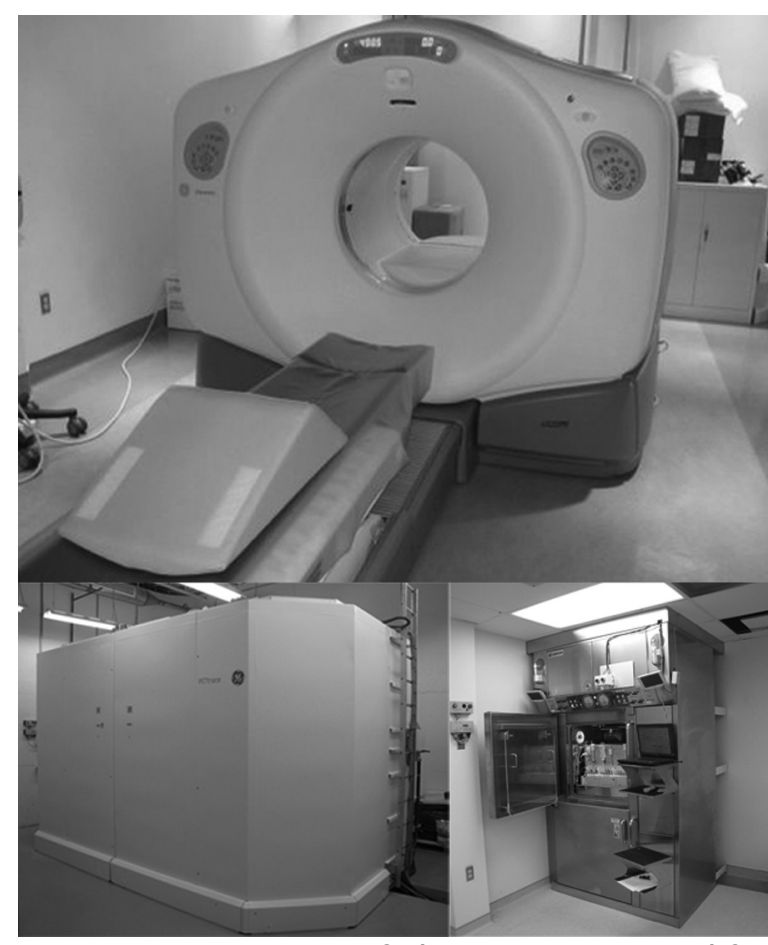

Figure 1. Components of the PET suite in Halifax including the PET-CT (top), cyclotron which produces the radioisotope ${ }^{18} \mathrm{~F}$ (bottom left) and the hot cell which produces ${ }^{18} \mathrm{~F}-\mathrm{FDG}$ from the ${ }^{18} \mathrm{~F}$ (bottom right).

PET are radioisotopes of fluorine $\left({ }^{18} \mathrm{~F}\right)$, oxygen $\left({ }^{15} \mathrm{O}\right)$, carbon $\left({ }^{11} \mathrm{C}\right)$ and nitrogen $\left({ }^{13} \mathrm{~N}\right)$. They are small organic elements that are readily incorporated into organic tracers. Their drawback is their short half-life, with ${ }^{18} \mathrm{~F}$ having the longest at 110 minutes, and ${ }^{15} \mathrm{O}$ having the shortest at 2 minutes. With these radioisotopes' short half-lives, they must either be produced on site (which requires a medical cyclotron) or, in the case of ${ }^{18} \mathrm{~F}$ with its 110 minute half life, production can occur at an offsite facility if delivery is possible within about 4 hours. With its recent installation of a cyclotron and radiopharmacy, the PET suite in Halifax can potentially use these radiopharmaceuticals with shorter half-lives.

The most widely used radiopharmaceutical in PET is ${ }^{18}$ fluoro-2-deoxy-D-glucose ( $\left.{ }^{18} \mathrm{~F}-\mathrm{FDG}\right)$, a glucose analog labeled with ${ }^{18} \mathrm{~F}$. It is used in the vast majority of clinical oncology and neurology applications, as well as a substantial portion of cardiac PET studies. However, ${ }^{18} \mathrm{~F}$ and the other PET radioisotopes can also be incorporated into other organic molecules to be used as tracers to exploit alternate metabolic pathways, both clinically and in research.

\section{PET-CT}

A PET-CT scanner is a PET scanner integrated with a multi-slice CT scanner. This allows for better localization as it produces both anatomical and molecular images. The CT also employs attenuation

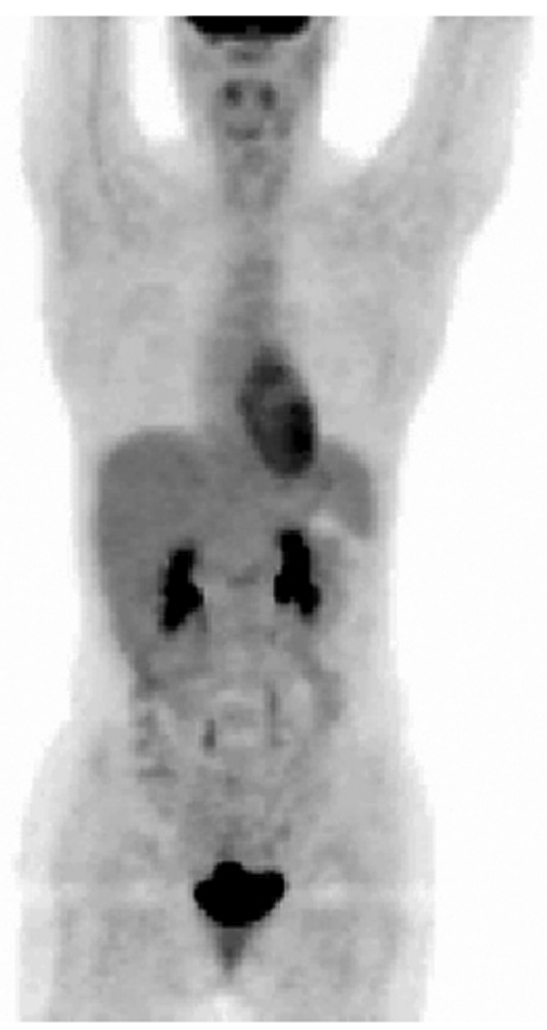

Figure 2. A normal ${ }^{18} \mathrm{~F}-\mathrm{FDG}$ PET scan depicting the normal bio-distribution of ${ }^{18} \mathrm{~F}-\mathrm{FDG}$. In the brain there is intense uptake, with gray matter having more uptake than white matter. The heart has variable uptake depending on its metabolic demand. Due to FDG excretion by the kidneys, there is also increased uptake in the urinary system with the most intense uptake in the renal collecting systems, the ureters, and in the urinary bladder. Throughout the GI tract there can be variable uptake. Mild uptake is present in muscles and bone marrow.

correction, which compensates for greater absorption in the center of the body as opposed to the peripheries. The combination of these two technologies has been so successful that it is now the standard.

\section{PET in Halifax}

The first PET-CT study in Halifax was performed on June 15, 2008 and, until recently, isotope was flown in daily from Montreal. However, with the recent completion of the PET suite in July 2010, the cyclotron and pharmacy is now producing ${ }^{18} \mathrm{~F}-\mathrm{FDG}$ on site. Demand for PET-CT studies continues to increase. It is currently only funded for oncology studies in Nova Scotia, but the hope is to expand this to the areas of cardiology and neurology.

\section{PET in Oncology}

In the past few years PET has emerged as a very useful imaging tool in oncology. While PET is a high-end modality that comes with a significant cost, this cost 
can be offset through better staging and diagnosis. As compared to CT, which demonstrates anatomy, PET displays physiology and metabolism. Compared with normal cells, cancer tissue may have increased glycolysis, protein synthesis, DNA synthesis, amino acid transport and blood flow, along with more anoxic or hypoxic cells. A variety of PET radiopharmaceuticals have been developed to image these deviations from normal physiological cell states, allowing PET to detect most forms of cancer. The vast majority of clinical PET oncology imaging is performed using ${ }^{18} \mathrm{~F}-\mathrm{FDG}$, which provides an image of tissue glycolysis. ${ }^{18} \mathrm{~F}$-FDG behaves in most respects like glucose, entering cells via glucose transporters and being phosphorylated via the enzyme hexokinase. However, unlike glucose, it cannot proceed further along the glycolytic pathway, and remains in the cell. Although ${ }^{18} \mathrm{~F}-\mathrm{FDG}$ also enters normal cells, there is substantially greater ${ }^{18} \mathrm{~F}$-FDG uptake in cancer cells, due to increased glucose transporters. There is also an increase in the enzyme hexokinase, and a decrease in phosphatase, not allowing glucose and ${ }^{18} \mathrm{~F}-\mathrm{FDG}$ to exit the cell.

PET provides added value over conventional imaging alone in a number of stages in the management of oncology patients. In cancer diagnosis, PET can be an effective indicator of benign versus malignant lesions. This is particularly relevant in the evaluation of the solitary pulmonary nodule, as discussed later. By imaging the whole body, PET leads to improvements in the staging of cancer, including local tissue involvement and the presence of distal metastases. In conjunction with CT, PET contributes to radiation therapy planning. Finally, the ability of PET to detect cancerous lesions makes it an effective tool for monitoring response to treatment and for subsequent follow-up of cancer patients.

The emergence of PET as an alternate imaging modality has led to an average $30 \%$ change in patient management across all cancer imaging, ${ }^{1}$ including $37 \%$ changes in lung and colorectal cancer, 33\% in head and neck cancer, $23 \%$ in lymphoma, 23\% in esophageal cancer, $29 \%$ in melanoma, and $28 \%$ in breast cancer. While PET plays a role in many different areas of oncology, this article will review the use of PET in 4 of the most common malignancies

\section{Lung Cancer}

Lung cancer is the leading cancer related cause of death in Western society. A possible reason for this disappointing figure is that lung cancer is usually a late diagnosis. ${ }^{2}$ To date, CT has been the leading imaging modality used to diagnose and stage lung cancer. The primary curative treatment for lung cancers is surgical resection, but this therapy can lead to futile surgeries in benign or metastatic cancers. By using PET for lung cancer imaging, a more accurate diagnosis and staging may be obtained, reducing these futile surgeries.

CT can be a useful tool in detecting benign versus malignant tumours; however, the diagnosis is made from anatomical characteristics alone. PET has been shown to differentiate benign versus malignant tumors in solitary pulmonary nodules based on FDG uptake. In one meta-analysis, the sensitivity and specificity of FDG-PET in diagnosing focal pulmonary lesions of $\geq 1 \mathrm{~cm}$ was found to be $97 \%$ and $78 \%$ respectively. Even though PET does demonstrate a relatively high sensitivity and specificity in diagnosing lung cancer, there are certain diseases that can hinder an accurate diagnosis. False-positives can occur in patients with tuberculosis and histoplasmosis and false-negatives in

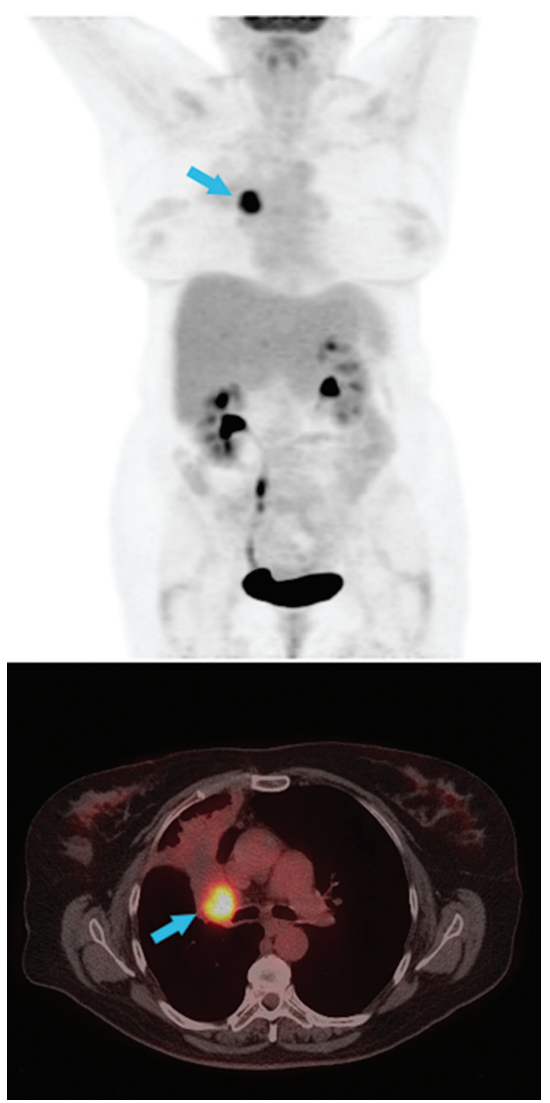

Figure 3. A female with non-small cell lung cancer was found to have a primary mass in the right upper lobe as well as bilateral mediastinal nodes on CT (not shown). Images from PET-CT (top: anterior view from PET, bottom: axial slice of fused PET and CT data at the level of the lesion) show intense uptake in the primary (blue arrows) but no uptake in either lymph node, resulting in a change in staging and management. 


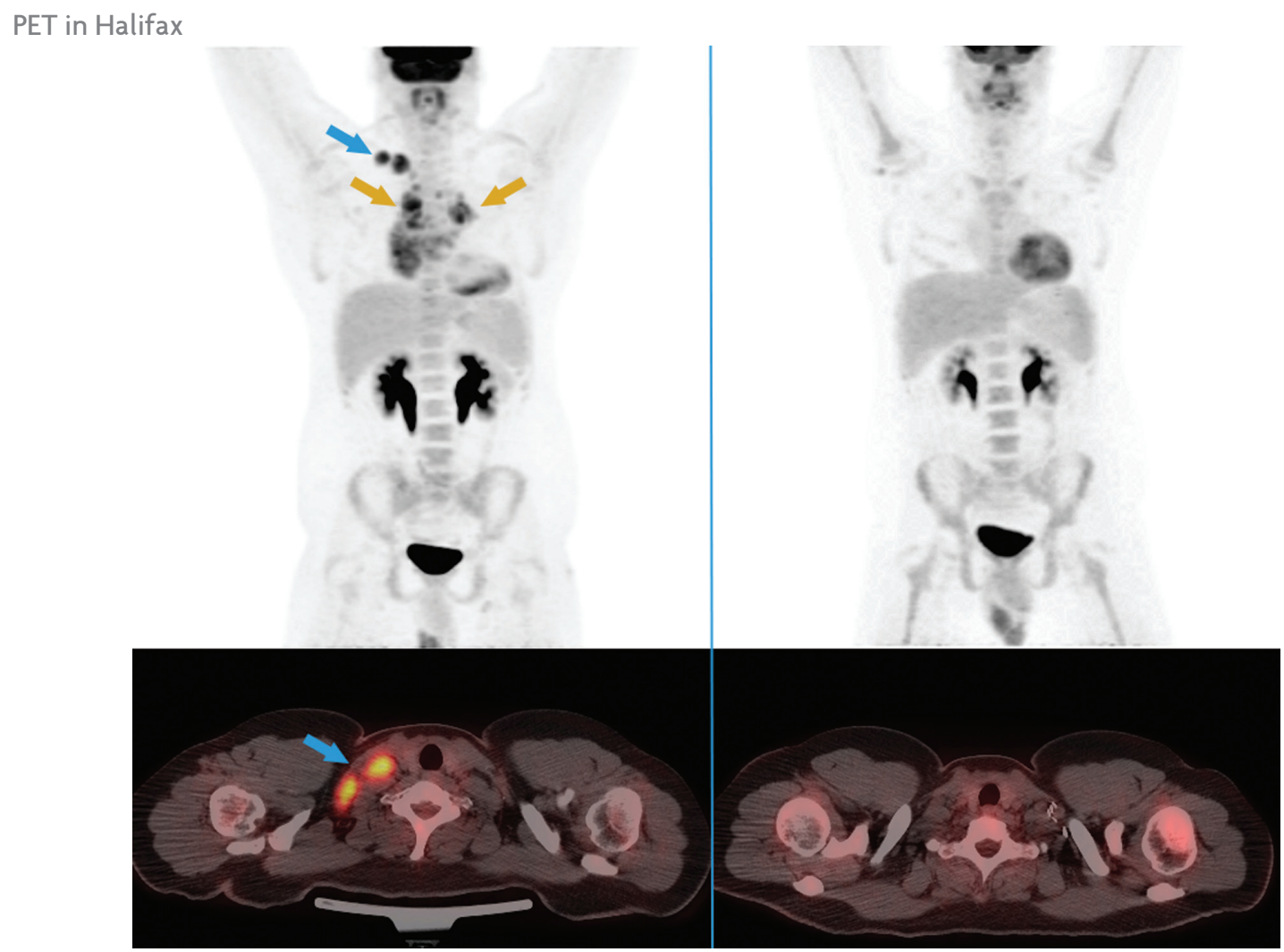

Figure 4. A young man with Hodgkin's Lymphoma. Initial CT (not shown) showed extensive mediastinal adenopathy but no disease outside of the mediastinum. Images (left) from a staging PET-CT (top: anterior PET image, bottom: axial fused PET and CT data) showed increased uptake in both supraclavicular (blue arrows) and mediastinal nodes (orange arrows). Images (right) taken from a follow up PET-CT show a complete metabolic response to therapy. Uptake low in the chest on the left is simply physiologic uptake in the heart at both time points, while increased generalized bone marrow uptake is typical post-chemotherapy.

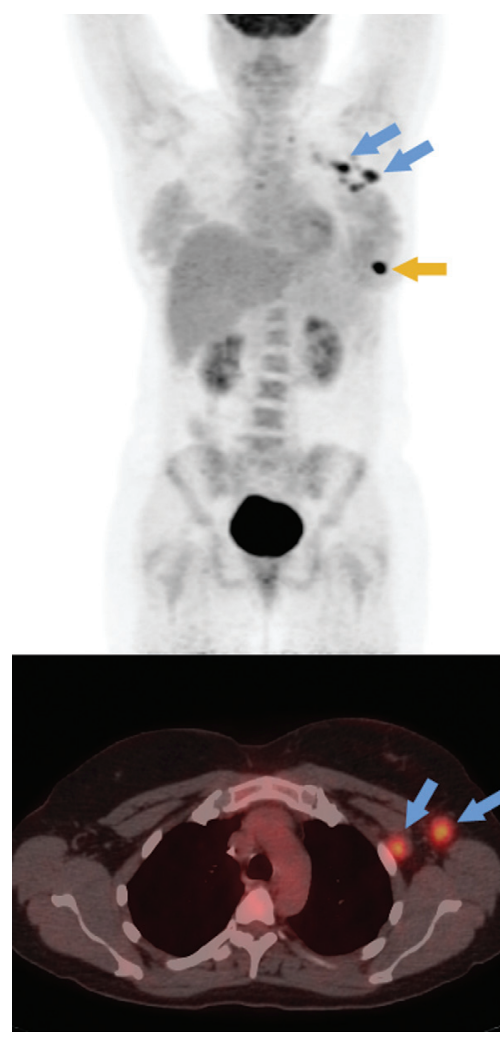

Figure 5. A female with breast cancer underwent PET-CT scanning to assess for distal metastases. The scan showed the primary (orange arrow) and involvement of axillary, supraclavicular, and subpectoral lymph nodes (blue arrows), but no distal metastases.

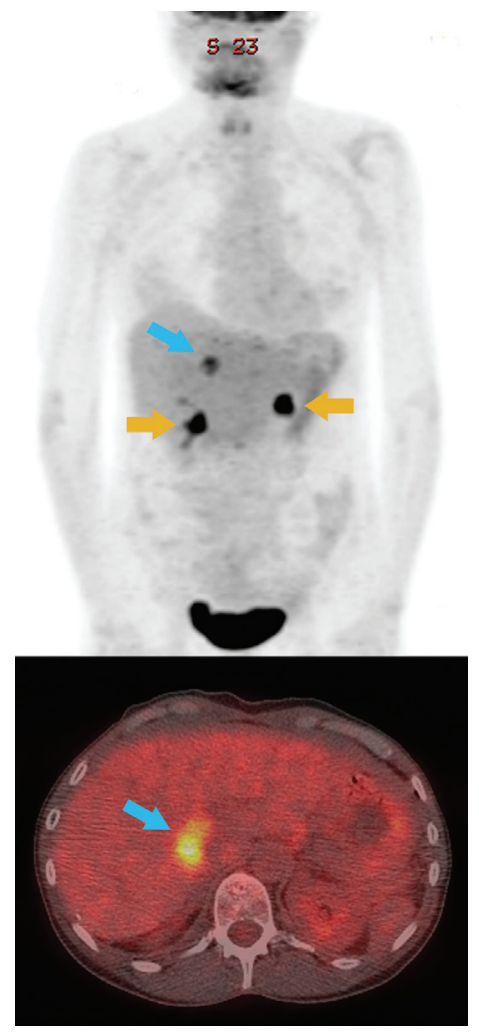

Figure 6. This patient with prior colorectal cancer was found to have rising tumor marker levels. Conventional imaging was inconclusive, however PET showed a solitary metastasis in the liver (blue arrows), allowing proper therapy to be delivered. Note is made of intense uptake in both kidneys (yellow arrows) which is physiologic. 
malignances less than $6 \mathrm{~mm}$ and bronchioloalveolar carcinomas. ${ }^{4}$

One of the greatest advantages PET has over CT is in the staging of lung cancer. In staging mediastinal cancers, PET has a pooled sensitivity and specificity of $84 \%$ and $89 \%$ respectively, while CT has a pooled sensitivity of $57 \%$ and specificity of $82 \% .{ }^{5}$ By using PET to stage lung cancers, it has modified the treatment plan in $60 \%$ of patients, including high impact changes in $35 \% .{ }^{6}$ One of the primary roles of lung cancer staging is to determine if the tumor is curative through surgery. Patients considered for surgery include those with stage I-II or stage IIIA. ${ }^{7}$ PET has frequently detected distant metastases in $5-10 \%$ of these patients with the most common sites being bone, liver and adrenal gland. ${ }^{8}$ In preoperative patients, new PET findings usually result in up-staging rather than down-staging, with the overall impact being less aggressive surgery and, more importantly, a reduction in futile thoracotomies from $41 \%$ to $21 \%{ }^{9}$

PET is also useful in follow-up imaging after treatment to determine the viability of previously treated tumors. A recurrence of the tumor may be missed with conventional imaging because changes in tumor size and appearance may be due to scar tissue or necrosis. PET can be used to detect changes in tumor metabolism after treatment to determine the presence of residual malignancy in the treated mass. This information can be used for therapy monitoring and assessing for recurrence.

\section{Lymphoma}

The evaluation of patients with lymphoma includes physical examination, laboratory data, bone marrow biopsy, and imaging. CT has been the accepted imaging modality for Hodgkin's Lymphoma (HD) and non-Hodgkin's Lymphoma (NHL) and is the basis of the Cotswold staging classification for $\mathrm{HD} .{ }^{10} \mathrm{CT}$ evaluation for the presence of malignancy in lymphoma is based mainly on size criteria. PET, with its ability to functionally image lymph nodes, has an obvious advantage in the evaluation of malignancy. Further, the sensitivity of CT for extra nodal involvement is not optimal, as CT may miss peripheral sites which may be identified on PET due to the high tumor-to-background activity on PET. CT may also provide images in which it is difficult to distinguish between a malignant and fibrotic tissue following therapy. ${ }^{11}$

Obtaining the correct staging of the disease is crucial for determining the proper patient treatment and prognosis. PET is well suited for whole body imaging and is considerably more useful in staging and assessing follow-ups. The use of PET in initial staging of HD up-staged $28.8 \%$ of the patients as compared to other imaging modalities and only $2.7 \%$ of the patients were down-staged. ${ }^{12}$ The results indicate that PET use in initial staging and diagnosis can impact the expected prognosis of patients with HD. In a meta-analysis of twenty studies that looked at the diagnostic performance of PET in staging lymphoma, PET was found to have a median sensitivity of $90.3 \%$ and a median specificity of $91.1 \%{ }^{13}$ The pooled false-positive rate was $10.3 \%$ and appeared to be higher in those with HD as compared to those with NHL. These results indicate that PET is an effective tool for staging and re-staging patients with lymphoma. In a retrospective study, it was found that of 1537 anatomical sites assessed, 48 of those sites had inconsistent PET and CT findings. Of those 48 sites, PET findings were correct in $83 \%$ (31 positive) of the cases, whereas CT had only five correct findings. ${ }^{14}$ Furthermore, PET gave the correct staging in 9 of the 53 total patients where CT staging was incorrect.

PET has shown to have better sensitivity and specificity than CT in staging lymphoma. PET scans are occasionally used in the radiation planning process. In a retrospective comparison of PET and CT use to assess the radiation therapy planning process, Lee et al. $(2004)^{15}$ found that in $10 / 17$ patients with positive PET and CT findings, the gross tumor volume was found to be smaller on PET in six of the cases. Consequently, the use of PET in radiation planning may reduce the subjectivity of the radiation treatment dose, potentially altering patient management.

PET can also be a useful tool in following post-treatment responses in patients with HD. In 28 patients following treatment for HD, CT had a sensitivity of $25 \%$ and a specificity of $42 \%$ whereas PET had a sensitivity and specificity of $100 \%$ and $83 \%$ respectively. ${ }^{16}$

\section{Breast}

Breast cancer is the most common female cancer in the US and the second most common cause of death in women. ${ }^{17}$ Although it is curable if detected early enough, approximately one third of the patients diagnosed with breast cancer will die of the disease.$^{18}$ Mammography is the imaging modality routinely used to detect breast cancer and while the sensitivity of mammography is excellent, the specificity is relatively low because of high false-positives. ${ }^{19,20}$ While not indicated in routine screening due to its high rate of false-negatives, especially in tumours $<10 \mathrm{~mm},{ }^{21}$ primary breast cancers visualized on PET may reveal important prognostic factors. A high degree of ${ }^{18} \mathrm{~F}-\mathrm{FDG}$, for example, has 
been shown to correlate with histologic grade and cell proliferation indices. ${ }^{22} \mathrm{PET}$ also shows a low sensitivity for detecting metastatic spread to lymph nodes. However, with its high specificity (96\% in one study), a positive PET scan may obviate the need for invasive lymph node dissection. ${ }^{23}$

PET's main application in breast cancer is in distal staging and evaluating for recurrence. Clinical response is determined after several cycles of chemotherapy by measuring changes in tumor size as assessed by conventional imaging procedures including $\mathrm{CT}$, MRI, plain film radiography, or ultrasound. A major advantage of PET imaging compared with conventional imaging is that it scans the entire patient for local recurrence, lymph node metastases and distant metastases during a single whole-body examination. PET has a reported sensitivity and specificity of $85.2 \%$ and $82.6 \%$ respectively in patients with breast cancer after neo-adjuvant chemotherapy. ${ }^{24}$ In 25 women with a suspected relapse of breast cancer, PET showed increased uptake in 43 areas, 22 correctly confirming the area of suspected relapse and 21 indicating other sites of metastases. Compared with conventional imaging, PET revealed additional lesions in two women with primary cancers and three with relapse, changing patient management for five women. ${ }^{25}$

\section{Colorectal}

The incidence of colorectal cancer in Western society is approximately $12-13 \%$, making it one of the most common types of cancer. Colorectal carcinoma is the third most common malignancy in men, second in females and fourth leading cause of cancer death. ${ }^{26}$ When diagnosed early, surgical treatment is frequently curative with minimal morbidity and mortality rates.

Colonoscopy and barium enemas are the standard modes of detection of primary colorectal carcinoma. PET has been used as an effective imaging method for recurrent colorectal cancer, but its role in detecting primary lesions has not yet been established. In a prospective study of 45 patients with colonic neoplasms, PET had a sensitivity of $62 \%$. PET only dectected $14 \%$ of protruded premalignant lesions between 1 and 1.9 $\mathrm{cm}, 17 \%$ of cancers smaller than $2 \mathrm{~cm}$ and only $23 \%$ of flat premalignant lesions. However, PET did detect $100 \%$ of all cancers greater than $2 \mathrm{~cm} .{ }^{27}$

PET is primarily used in the detection and localization of recurrent cancer in patients with rising carcinoembryonic antigen (CEA) levels and it can also be helpful in contributing to surgical decision making. In a comparison of one hundred whole-body PET scans of patients with colorectal cancer with CT, liver ultrasound, and a carcinoembryonic antigen test, PET had a sensitivity of $98 \%$ and a specificity of $90 \%$. The respective sensitivity and specificity for CT was $91 \%$ and $72 \%$ and for the CEA test $76 \%$ and $90 \%$. For the detection of liver metastases PET had a sensitivity of $100 \%$ and a specificity of $99 \%$ whereas ultrasound had a sensitivity of $87 \%$ and specificity of $96 \%{ }^{28}$

\section{PET in Neurology}

Dementia is a neurodegenerative disease that can have a tremendous impact on the patient, their family and society in general. Management of patients with dementia relies on early recognition and accurate assessment of cognitive and behavioral symptoms. Through the combination of history and physical exam, laboratory tests and structural neuroimaging (CT and MRI), an appropriate management plan may be implemented. Over the past two decades studies have shown that neurodegenerative diseases can produce alterations in brain metabolism that can be measured with PET. ${ }^{29}$ PET can also distinguish normal degenerative changes due to aging versus dementia, and it can be used to assess very early stages of the disease. In a study of 284 patients undergoing evaluation for dementia with PET studies, progressive dementia was found with a sensitivity and specificity of $93 \%$ and $76 \%$ respectively. Regional brain metabolism is a sensitive indicator of Alzheimer's dementia (AD) and neurodegenerative diseases in general. ${ }^{31}$

Considerable data exists that supports the idea that the pattern of metabolism and perfusion abnormalities in $\mathrm{AD}$ is quite different from frontal lobe dementia, which is characterized by reduced frontal lobe metabolism and perfusion. ${ }^{31}$ In $\mathrm{AD}$, there is temporoparietal hypometabolism. ${ }^{32}$ Dementia with Lewy Bodies is also clinically different than $\mathrm{AD}$, displaying temporoparietal hypometabolism along with abnormalities in the visual association cortex of the occipital lobe. ${ }^{33}$

Complex partial seizures remain uncontrolled in a significant proportion of patients despite medical therapy. Surgical removal of the epileptogenic foci in partial seizures results in significant improvement in control of the seizures ${ }^{34}$ MRI is capable of detecting the source of the seizures in the majority of patients with partial seizures. However, about $20-30 \%$ of the patients with focal epilepsy have a normal MRI. The main clinical use of PET in epilepsy is the localization of the epileptogenic foci in surgical candidates with partial seizures and combining this with other investigational modalities such as electroencephalography. In partial seizures, there is an increase in glucose metabolism and 
cerebral blood flow in the region of the epiletogenic foci during the ictal period. ${ }^{35}$ Due to the difficulties in injecting and imaging during the ictal period, most studies are done during the inter-ictal period when the area of interest actually shows hypometabolism on PET imaging.

Clinical detection of the various movement disorders can be very difficult, especially in the early stages. Furthermore, MRI often reveals no structural abnormalities. FDG PET in Parkinson's disease shows normal to increased glucose metabolism in the striatum, but decreased metabolism in the temporoparietal areas. ${ }^{36}$ More novel radiopharmaceuticals have also been used. In particular, ${ }^{18} \mathrm{~F}$-DOPA has shown promise in the evaluation of Parkinson's disease.

\section{PET in Cardiology}

Cardiac nuclear imaging is performed on a regular basis with traditional (non-PET) radiopharmaceuticals and imaging with a single photon emission computed tomography (SPECT) camera. Although similar, cardiac imaging with PET offers potentially better capabilities. The two main applications are in assessing myocardial perfusion and myocardial viability.

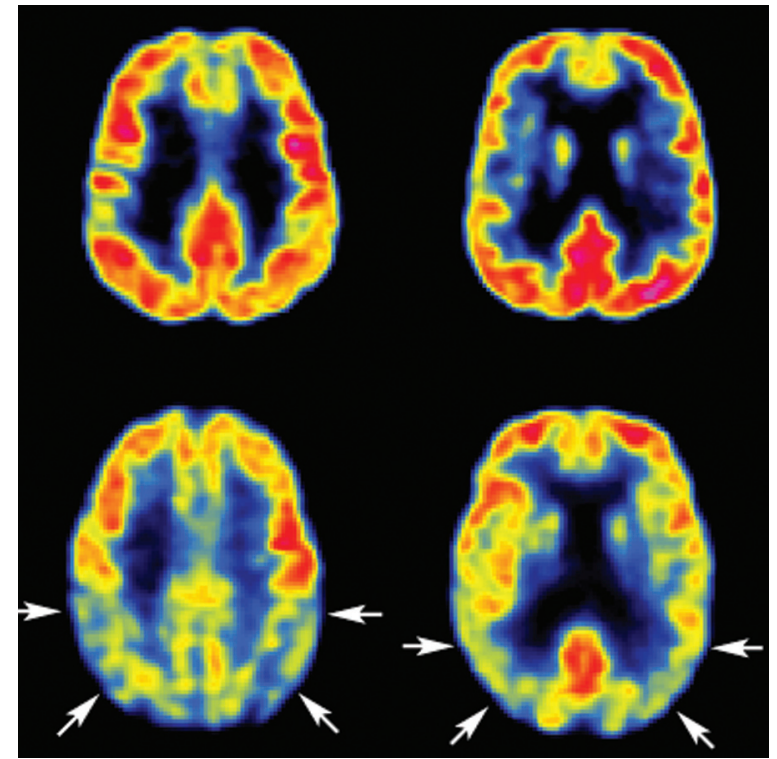

Figure 7. PET images of the brain. The top row demonstrates normal brain metabolism. The bottom two images were taken from a patient with Alzheimer's dementia and show marked hypometabolism of the parietal lobe bilaterally (arrows).

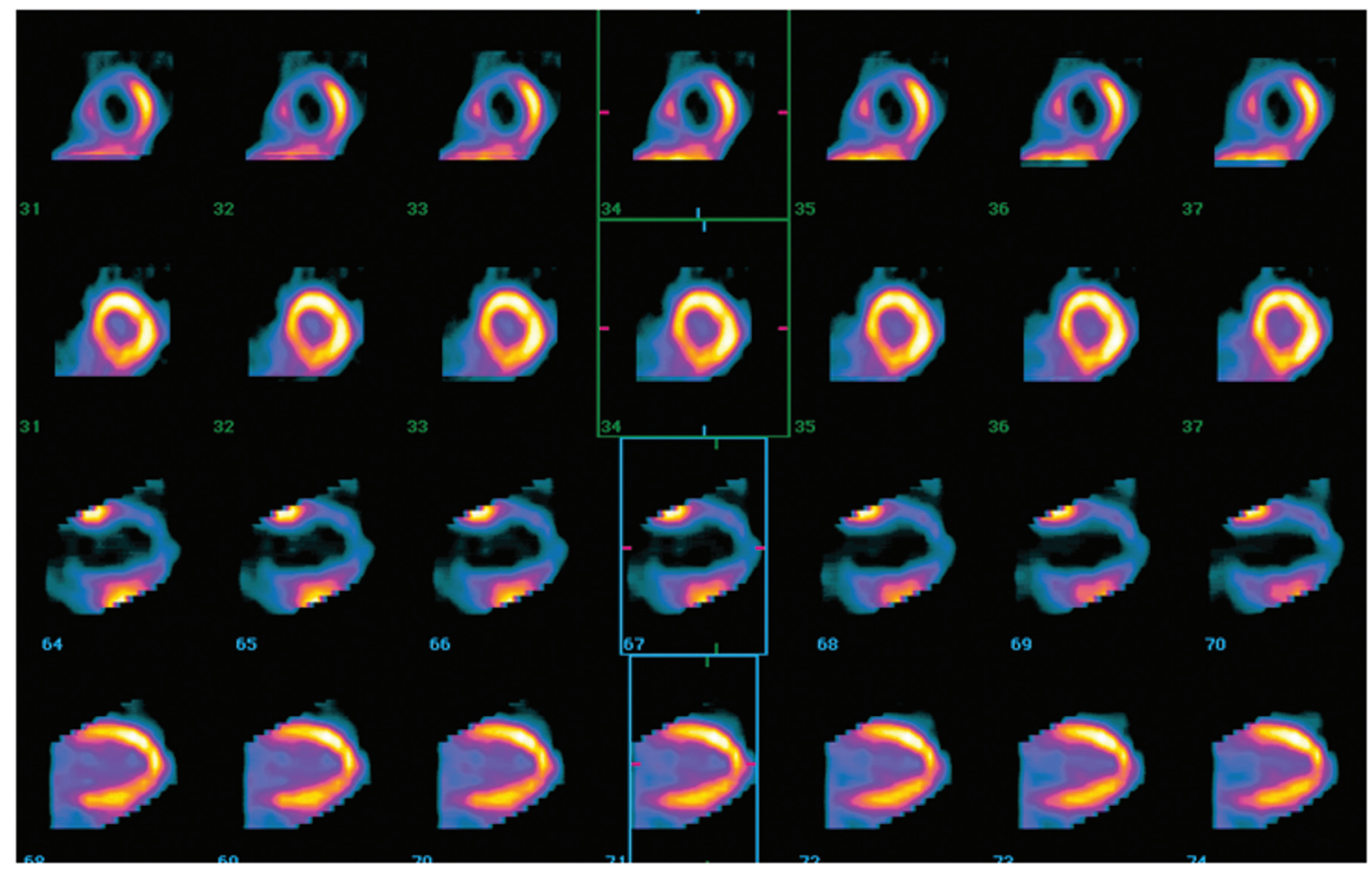

Figure 8. PET images of the heart. Top two rows are short axis views through the left ventricle; bottom two rows are long axis views. In both sets, the upper row is a PET perfusion study using $13 \mathrm{~N}$-ammonia, while the lower row is a PET metabolism study using ${ }^{18} \mathrm{~F}-\mathrm{FDG}$. There are extensive, severe, perfusion abnormalities, but these areas show normal ${ }^{18}$ F-FDG uptake and hence are viable. Thus, the patient will likely benefit from a revascularization procedure. 
With respect to myocardial perfusion, the PET approach offers the potential to measure absolute coronary blood flow and coronary blood flow reserves, while SPECT only shows relative regional myocardial perfusion. Further advantages of PET include better resolution and accurate attenuation correction. When compared with the gold standard of invasive coronary angiography, cardiac PET, using $31 \mathrm{~N}$ ammonia and ${ }^{18} \mathrm{RB}$, has demonstrated an overall sensitivity of $93 \%$ and specificity of $92 \%$ when diagnosing coronary artery disease. ${ }^{37}$ In comparison to SPECT, Go and colleagues showed a $95 \%$ sensitivity and $82 \%$ specificity for $82 R B$ PET compared to a $79 \%$ and $76 \%$ respectively for SPECT. ${ }^{38}$

In assessing myocardial viability, the intent is to determine whether areas of poorly perfused myocardium are still alive and hence may benefit from revascularization. Viability may be assessed with SPECT agents, MRI, dobutamine echo, or PET, with PET generally considered the gold standard. Viable myocardium metabolizes glucose, and hence viability assessment with PET is predicated on demonstrating ${ }^{18}$ F-FDG uptake in the areas of poorly perfused myocardium.

\section{Conclusion}

The impact of positron emission tomography on expected management of patients with cancer was recently studied by Hillner et al. They found that PET was associated with a change in management for $43.1 \%$ of patients. ${ }^{39}$ It has also been consistently demonstrated that PET finds more sites of active disease than CT or other imaging modalities. PET is a powerful tool in the management of cancer patients and shows significant promise in many other fields including neurology and cardiology.

\section{References}

1. Gambhir SS, Czernin J, Schwimmer J, Silverman DH, Coleman RE, Phelps ME. A tabulated summary of the FDG PET literature. J Nucl Med 2001;42:1S-93S.

2. Van Zandwijk N. New methods for early diagnosis of lung cancer. Lung Cancer 2002; 38(1):S9-11.

3. Gould M, Maclean C, Kushner W, et al. Accuracy of positron emission tomography for diagnosis of pulmonary nodules and mass lesions: a meta-analysis. JAMA 2001; 285(7):914-924.

4. Ohtsuka T, Nomori H, Watanabe K, Naruke T. False-positive findings on FDG-PET caused by non-neoplastic cellular elements after neoadjuvant chemoradiotherapy for non-small cell lung cancer. Jap J Clin Oncol 2005; 35(5):272-273.

5. Toloza EM, Harpole L, McCrory DC. Noninvasive staging of nonsmall cell lung cancer: a review of the current evidence. Chest. 2003;Jan;123:137S-146S.

6. Hicks RJ, Kalff V, MacManus MP, Ware RE, McKenzie AF,
Matthews JP, Ball DL. The utility of (18)F-FDG PET for suspected recurrent non-small cell lung cancer after potentially curative therapy: impact on management and prognostic stratification. J Nucl Med. 2001;42(11):1605-13.

7. Passlick B. Initial surgical staging of lung cancer. Lung Cancer. 2003;42:S21-5.

8. Mac Manus MP, Hicks RJ, Matthews JP, McKenzie A, Rischin D, Salminen EK, Ball DL. Positron emission tomography is superior to computed tomography scanning for response-assessment after radical radiotherapy or chemoradiotherapy in patients with nonsmall-cell lung cancer. J Clin Oncol. 2003;21(7):1285-92.

9. van Tinteren H, Hoekstra OS, Smit EF, van den Bergh JH, Schreurs AJ, Stallaert RA, van Velthoven PC, Comans EF, Diepenhorst FW, Verboom P, van Mourik JC, Postmus PE, Boers M, Teule GJ. Effectiveness of positron emission tomography in the preoperative assessment of patients with suspected non-smallcell lung cancer: the PLUS multicentre randomised trial. Lancet. 2002;359(9315):1388-93.

10. Crowther D, Lister R. The Cotswold report on the investigation and staging of Hodgkin's disease. Br J Cancer 1990;62:551-552.

11. Bangeter M, Grisshammer M, Bergmann L. Progress in medical imaging of Lymphoma and Hodgkin's disease. Curr Opin Oncol 1999;11:339-342.

12. Munker R, Glass J, Griffeth LK, Sattar T, Zamani R, Heldmann M, Shi R, Lilien DL. Contribution of PET imaging to the initial staging and prognosis of patients with Hodgkin's disease. Ann Oncol 2004;15:1699-1704.

13. Isasi CR, Lu P, Blaufox MD. A metaanalysis of 18F-2-deoxy-2fluoro-D-glucose positron emission tomography in the staging and restaging of patients with lymphoma. Cancer. 2005;104(5):106674.

14. Tatsumi M, Cohade C, Nakamoto Y, Fishman EK, Wahl RL. Direct comparison of FDG PET and CT findings in patients with lymphoma: initial experience. Radiology. 2005;237(3):1038-45.

15. Lee YK, Cook G, Flower MA, Rowbottom C, Shahidi M, Sharma B, Webb S. Addition of 18F-FDG-PET scans to radiotherapy planning of thoracic lymphoma. Radiother Oncol. 2004;73(3):27783.

16. Rigacci L, Castagnoli A, Dini C, Carpaneto A, Matteini M, Alterini R, Carrai V, Nassi L, Bernardi F, Pieroni C, Bosi A. 18FDGpositron emission tomography in post treatment evaluation of residual mass in Hodgkin's lymphoma: long-term results. Oncol Rep 2005;14(5):1209-14.

17. Jemal A, Siegel R, Ward E, Hao Y, Xu J, Thun MJ. Cancer statistics 2009. CA Cancer J Clin. 2009;59(4):225-49.

18. Greenlee RT, Hill-Harmon MD, Murray T. Cancer statistics. CS Cancer J Clin 2001; 51:15-56.

19. Rankin SC. MRI of the breast. Br J Radiol 2000;73:806-818.

20. Saarenma I, Salminen T, Geiger V et al. The Visibility of cancer on earlier mammograms in a population-based screening programme. Eur J Cancer 1999; 35:1118-1120.

21. Kumar R, Chauhan A, Zhuang H, Chandra P, Schnall M, Alavi A. Clinicopathologic factors associated with false negative FDG-PET in primary breast cancer. Breast Cancer Res Treat 2006;98(3):267-274.

22. Oshida M, Uno K, Suzuki M, et al. Predicting the prognoses of breast carcinoma patients with positron emission tomography using 2-deoxy-2-fluoro [18F]-D-glucose. Cancer 1998;82:22272234 .

23. Dehdashti F. PET in Breast Cancers. In: Wahl RL, ed. Principles and Practice of PET and PET/CT, 2nd ed. Philadelphia: Lippincott Williams \& Wilkins, 2002:287-309.

24. Kim SJ, Kim SK, Lee ES, Ro J, Kang S. Predictive value of [18F] FDG PET for pathological response of breast cancer to neoadjuvant chemotherapy. Ann Oncol. 2004;15(9):1352-7.

25. Landheer ML, Steffens MG, Klinkenbijl JH, Westenberg AH, Oyen WJ. Value of fluorodeoxyglucose positron emission tomography 
in women with breast cancer. Br J Surg 2005;92(11):1363-7.

26. Landis SH, Murray T, Bolden S, Wingo PA. Cancer Statistics 1999. CA Cancer J Clin 1999; 49:8-31.

27. Friedland S, Soetiko R, Carlisle M, Taur A, Kaltenbach T, Segall G. 18 Fluorodeoxyglucose positron emission tomography has limited sensitivity for colonic adenoma and early stage colon cancer. Gastrointest Endosc 2005;61(3):395-400.

28. Staib L, Schirrmeister H, Reske SN, Beger HG. Is (18) F-fluorodeoxyglucose positron emission in recurrent colorectal cancer a contribution to surgical decision making? Am J Surg. 2000;180(1):1-5.

29. Siverman DH. Brain 18F-FDG PET in the diagnosis of neurodegenerative dementias: comparison with perfusion SPECT and with clinical evaluations lacking nuclear imaging. Journal of Nuclear Medicine. 2004;45(4):594-607.

30. Silverman DH, Small GW, Chang CY, Lu CS, Kung de Aburto MA, Chen W, Czernin J et al. Positron emission tomography in evaluation of dementia: Regional brain metabolism and longterm outcome. JAMA 2001;286(17):2120-2127.

31. Santens P, De Bleecker J, Goethals P, Strijkmans K, Lemahieu I, Slegers G, et al. Differential regional cerebral uptake of (18)fluoro-2-deoxy-D-glucose in Alzheimer's disease and frontotemporal lobe dementia at initial diagnosis. Eur Neurol 2001;45:19-27.

32. Minoshima S, Frey KA, Koeppe RA, Foster NL, Kuhl DE. A diagnostic approach in Alzheimer's disease using threedimensional stereotactic surface projections of fluorine-18FDG PET. J Nucl Med 1995;36:1238-1248.

33. Lobotesis K, Fenwick JD, Phipps A, Ryman A, Swann A, Ballard $C$ et al. Occipital hypoperfusion on SPECT in dementia with Lewy bodies but not AD. Neurology 2001;56:643-649.

34. Wiebee S, Blume WT, Gavin JP et al. A Randomized controlled trial of surgery for temporal lobe epilepsy. N Eng J Med 2001;345:311-318.

35. Engel J Jr, Kuhl DE, Phelps ME. Regional brain metabolism during seizures in humans. Adv Neurol 1983;34:141-148.

36. Hu MT, Taylor-Robinson SD, Chaudhuri KR, et al. Cortical function in non-demented Parkinson's disease patients: a combined (31)P-MRS and (18)FDG-PET study. Brain 2000;123(Pt2):340-352.

37. Machac J. Cardiac positron emission tomography imaging. Sem Nucl Med 2005;35:17.

38. Go R, Marwick T, MacIntrye W, et al. A prospective comparison of rubidium-82 PET and thallium-201 SPECT myocardial perfusion imaging utilizing a single dipyridamole stress in the diagnosis of coronary artery disease. J Nucl Med 1990;31:18991905.

39. Hillner B, Siegel B, Shields A, Liu D, Gareen I, Hanna L, Stine $\mathrm{SH}$, Coleman RE. The impact of positron emission tomography (PET) on expected management during cancer treatment. Cancer 2008;115(2):410-418.

\section{Live, work and play on the South Shore}

Imagine yourself kayaking, canoeing, sailing, whale watching, diving or enjoying one of our many golf courses, hiking trails, theatres, galleries or museums. We are located on Nova Scotia's South Shore (one hour from metro Halifax), where miles of coastline are marked by beautiful, sandy beaches and picturesque towns including Bridgewater, Chester, Liverpool, Lunenburg and Mahone Bay; where hundreds of lakes, streams and rivers flow through small fishing villages and dense forests.

The District requires physicians for:

- Family Medicine

- Emergency Medicine

- Psychiatry

Candidates must be eligible for licensure in Nova Scotia.

Inquiries and applications may be directed to:

Dr. Peter Vaughan, VP Medicine

South Shore District Health Authority

90 Glen Allan Drive

Bridgewater NS B4V 3S6

Canada

Telephone: 902-527-5057

Fax: 902-527-5269

E-mail: pvaughan@ssdha.nshealth.ca

Website: www.ssdha.nshealth.ca

\section{Well pollnt \\ PHYSICIANS}

\section{Laval QC, Kanata Ottawa, Vaughan Ontario, Edmonton Alberta, and Coquitlam BC}
Wellpoint Health is one of Canada's fastest growing healthcare companies and requires physicians for family practice, and occupational medicine. We offer a turnkey operation for our physicians, utilization of EMR system, and offer
a competitve package that can include: large signing bonus, daily minimum guarantees, company paid vacations, or student load repayment plans!

\section{All Interested physicians contact:}

\section{Sunil Sharma}

Tel 647 637-2233

Email sunil.sharma@wellpointhealth.ca 